\title{
Comportement mécanique d'une paroi expérimentale renforcée par clouage
}

\author{
F. PELLET \\ P. EGGER \\ F. DESCCEUDRES \\ Institut des Sols, \\ Roches et Fondations \\ École Polytechnique \\ Fédérale de Lausanne
} CH 1015 Lausanne, Suisse
Une paroi expérimentale renforcée par clouage a été réalisée dans le cadre d'un chantier autoroutier. Cet ouvrage, de $10 \mathrm{~m}$ de hauteur, a été instrumenté de manière à suivre l'évolution des déplacements et des efforts mobilisés dans les clous au cours de sa réalisation. Les résultats indiquent que les déplacements sont concentrés dans une zone proche du parement et que les efforts, nettement inférieurs à la résistance des barres, varient en fonction de la nature des terrains traversés. Des calculs effectués par éléments finis montrent que l'on peut raisonnablement prédire le comportement de l'ouvrage.

\section{Mechanical behaviour of an experimental wall reinforced by nailing}

An experimental wall reinforced by soil nailing was built in the frame of highway works. This wall, of $10 \mathrm{~m}$ high, was instrumented in order to measure displacements and forces mobilised in the nails during the construction. The results show that displacements occur in the vicinity of the wall. Moreover forces in the nails which are lower than the tensile strength, depend on the mechanical characteristics of the encountered soils. A finite element analysis allows for a proper modelling of the mechanical behaviour of the wall. 


\section{Introduction}

Le développement croissant des infrastructures en site urbain associé à la nécessité de limiter l'emprise des ouvrages conduit fréquemment à assurer la stabilité des talus d'excavation par clouage du sol. Bien que ce procédé ait subi un essor considérable, le comportement mécanique des parois clouées n'est pas complètement éclairci.

Durant ces dernières années, les recherches se sont intensifiées pour tenter d'établir une description fidèle du comportement mécanique d'un massif cloué. Indépendamment des méthodes dites en déplacement comme la méthode des éléments finis, les approches actuelles reposent, pour la plupart, sur le principe du calcul à la rupture. On distingue les méthodes basées sur l'homogénéisation du milieu constitué par le sol et les clous (De Buhan, 1986; Anthoine, 1990) et celles qui se concentrent sur la description de l'interaction solclou au droit de la surface de rupture (Blondeau et al., 1984; Pellet, 1993).

La validation de ces méthodes de calcul doit nécessairement passer par la confrontation des résultats obtenus et des mesures réalisées sur des ouvrages judicieusement instrumentés, réalisés en vraie grandeur. Plusieurs ouvrages expérimentaux ont été réalisés notamment en France, dans le cadre du projet CLOUTERRE (Schlosser et al., 1993), et en Allemagne (Gudehus, 1981; Gässler, 1987).

Dans le cadre de la réalisation d'une tranchée couverte située sur le tracé de l'autoroute de contournement de la ville de Genève, le Département des travaux publics du canton de Genève a décidé la construction d'une paroi expérimentale renforcée par clouage. Cette étude (Pellet et Egger, 1994) vise à améliorer la compréhension du comportement de ce type d'ouvrage, dans le contexte géotechnique du bassin lémanique, en clarifiant le rôle joué par les clous et en tentant de répondre aux questions essentielles suivantes:

- Quels sont les déplacements horizontaux d'une paroi clouée?

- Quels sont les tassements en surface?

- Quelles sont les forces réellement mobilisées dans les barres?

\section{2}

\section{Présentation de l'ouvrage}

\section{Situation et caractéristiques géométriques}

L'ouvrage est une paroi clouée de $10 \mathrm{~m}$ de hauteur et de $32 \mathrm{~m}$ de longueur. La plate-forme, qui se situe à une cote altimétrique de $420,90 \mathrm{~m}$, a une largeur de $12 \mathrm{~m}$ au droit du profil P 250. Le parement de la paroi a un fruit de $1 / 10$ ( 1 horizontal pour 10 vertical), et le renforcement est assuré par six rangs de clous (Fig. 2).

En fin de réalisation, la plate-forme de l'ouvrage a été surchargée par environ $2 \mathrm{~m}$ de remblai mis en place progressivement. La figure 1 montre une vue générale de l'ouvrage terminé.

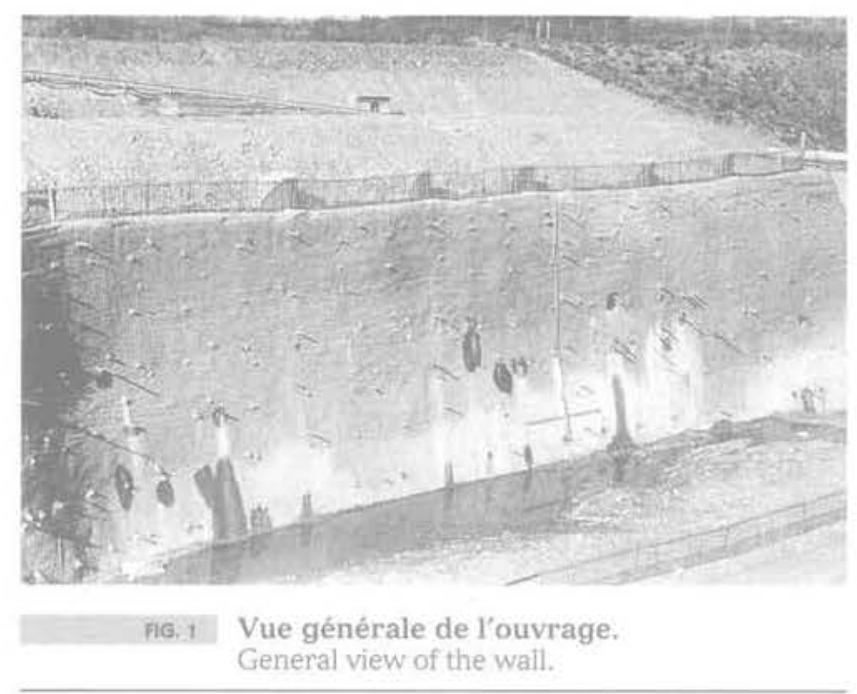

2.2

\section{Contexte géologique et hydrogéologique}

Comme l'essentiel du bassin genevois, le site a été modelé par la dernière grande glaciation de Würm. Dans la zone de l'ouvrage, la campagne de reconnaissance a permis de mettre en évidence trois formations distinctes dont les limites sont reportées à la figure 2.

La couche supérieure, peu cohérente et perméable, est constituée de cailloutis de retraits würmiens. Il s'agit de graviers de granulométrie moyenne se trouvant dans une matrice de sable gravillonneux de couleur gris beige. La couche intermédiaire est constituée de limon peu à moyennement argileux, souvent sableux, renfermant de nombreux cailloux de taille variable. Elle est relativement cohérente et imperméable. La couche inférieure dénommée "alluvion ancienne 1), est formée de cailloutis morainiques dans une matrice de sable plus ou moins limoneux renfermant des blocs épars de taille décimétrique. Cette formation qui peut être légèrement cimentée présente une assise de compacité élevée (Fig. 3). Du point de vue hydrologique, signalons que la nappe phréatique est très profonde et ne concerne pas la zone de l'ouvrage.

Plusieurs essais ont été réalisés in situ et en laboratoire sur des échantillons prélevés. L'analyse des résultats associée aux conclusions de la campagne de reconnaissance préliminaire réalisée pour le projet ont permis de définir les caractéristiques mécaniques des matériaux retenues pour les calculs de stabilité de l'ouvrage (tableau I).

\section{3}

\section{Réalisation de l'ouvrage}

L'ouvrage a èté exécuté d'avril à juin 1994. L'excavation, divisée en six étapes de hauteur variant entre $1,50 \mathrm{~m}$ et $2,25 \mathrm{~m}$, a été réalisée en continu sur toute la longueur de l'ouvrage, lorsque la tenue du terrain le permettait, et par plots alternés d'environ $5 \mathrm{~m}$ de longueur dans le cas contraire. La durée moyenne d'une étape d'excavation était d'environ une semaine.

Le parement est constitué d'une couche de béton projeté ayant une épaisseur théorique de $15 \mathrm{~cm}$. Il est armé par deux nappes de treillis soudés. 


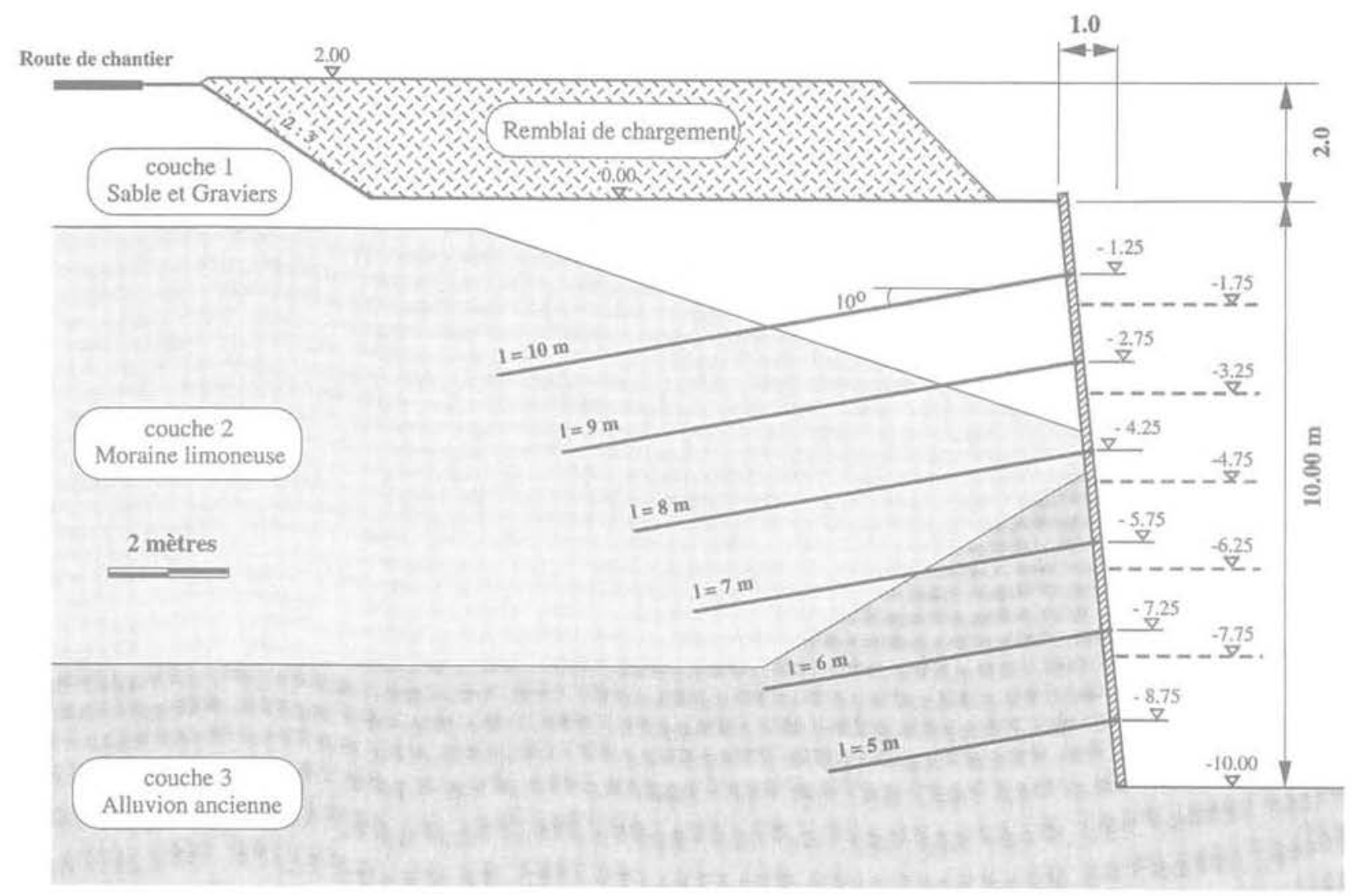

FiG. 2 Coupe transversale au profil 250.

Cross section at the profile 250 .

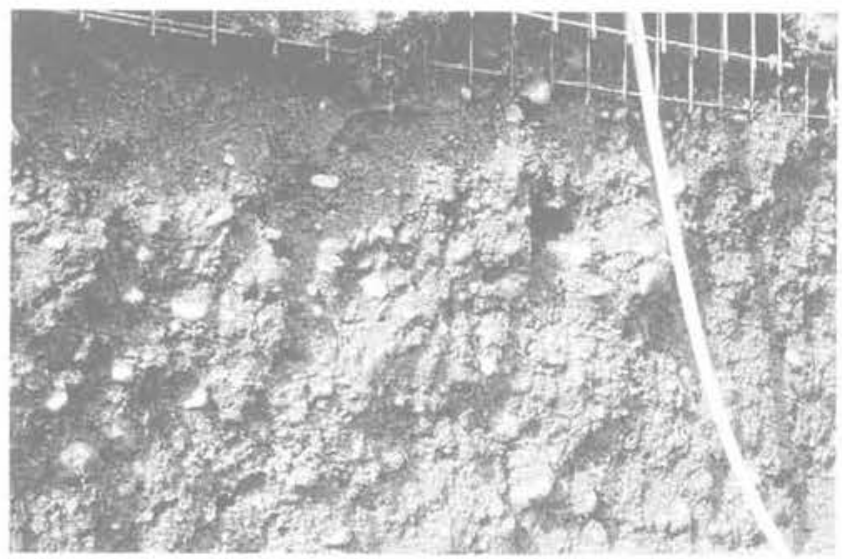

FG.3 Vue de la couche 3 .

View of the layer 3.
Le renforcement est assuré par six rangs de clous de longueur variant entre 5 et $10 \mathrm{~m}$ (Fig. 2). Ces clous sont disposés selon une maille rectangulaire, avec un espacement vertical de $1,5 \mathrm{~m}$ et un espacement horizontal de $2 \mathrm{~m}$. Les barres sont mises en place dans des forages de $114 \mathrm{~mm}$ de diamètre inclinés à 10 degrés, puis injectées gravitairement à l'aide d'un coulis de ciment. Deux jours après leur mise en place, elles sont réinjectées à l'aide d'un tube muni d'une manchette à son extrémité, puis serrées à la clé dynamométrique à une tension théorique de $50 \mathrm{kN}$.

Bien que la nappe phréatique soit située au-dessous de l'ouvrage, une batterie de drains subhorizontaux et de barbacanes a été mise en place pour évacuer d'éventulles poches d'eau.

A la fin de la réalisation de la paroi, une pause d'une semaine a été observée. Le remblayage de la plate-

TABLEAUI Caractéristiques mécaniques des géomatériaux pour les calculs de stabilité. Mechanical characteristics of geomaterials for stability analysis.

\begin{tabular}{c|c|c|c} 
& $\begin{array}{c}\text { Poids volumique } \\
\gamma\left[\mathrm{kN} / \mathrm{m}^{2}\right]\end{array}$ & $\begin{array}{c}\text { Cohésion } \\
\text { c [kPa] }\end{array}$ & $\begin{array}{c}\text { Angle de frottement } \\
\text { Ð degrés }\end{array}$ \\
\hline $\begin{array}{c}\text { Couche1 } \\
\text { Sable et graviers }\end{array}$ & 22,5 & $0-10$ & 33 \\
\hline $\begin{array}{c}\text { Couche2 } \\
\text { Moraine limoneuse }\end{array}$ & 23,5 & $10-50$ & 30 \\
\hline $\begin{array}{c}\text { Couche3 } \\
\text { Alluvion ancienne }\end{array}$ & 24,0 & $10-50$ & 40 \\
\hline
\end{tabular}


forme s'est ensuite opéré au rythme d'une couche de $70 \mathrm{~cm}$ d'épaisseur par semaine pour atteindre une hauteur de $2,10 \mathrm{~m}$. Le matériau utilisé est un mélange de sable et gravier provenant directement du chantier.

\section{4}

\section{Caractéristiques mécaniques des matériaux de construction}

Le béton projeté est dosé à $300 \mathrm{~kg} / \mathrm{m}^{3}$. Le coulis de scellement est composé d'un mélange d'eau et de ciment, dans un rapport $\mathrm{E} / \mathrm{C}$ égal à 1, et d'un adjuvant accélérateur de prise. Les essais en laboratoire, réalisés à 28 jours sur des éprouvettes cylindriques, ont indiqué les résistances à la compression simple suivantes:

- coulis $\sigma_{c, 28}=52 \mathrm{MPa}$

- béton projeté $\sigma_{c, 2 \mathrm{~B}}=18 \mathrm{MPa}$

Les clous sont des barres Swiss Gewi de $20 \mathrm{~mm}$ de diamètre dont les caractéristiques nominales sont les suivantes:

- diamètre $\mathrm{D}_{\mathrm{b}}=20 \mathrm{~mm}$

- limite élastique $\sigma_{\mathrm{et}}=500 \mathrm{MPa}$

- limite d'écoulement $\sigma_{e c}=600 \mathrm{MPa}$

- module d'élasticité $\mathrm{E}=210000 \mathrm{MPa}$

- déformation à la rupture $\varepsilon_{\mathrm{f}}=20 \%$

Quatre essais d'arrachement, dont l'un sur une barre instrumentée, ont été réalisés sur des clous de longueurs scellées variables, mis en place dans la couche de l'alluvion ancienne. Les résultats ont permis d'estimer la résistance du scellement à $70 \mathrm{kN}$ par mètre linéaire de barre, pour une longueur scellée d'environ $2 \mathrm{~m}$.

\section{5}

\section{Programme expérimental et dispositif d'auscultation}

Le programme expérimental a consisté à mesurer les déplacements horizontaux et verticaux (tassements) ainsi que les efforts mobilisés dans les barres au cours de la réalisation de l'ouvrage. Les mesures ont été faites après chacune des six étapes d'excavation et à l'issue des trois phases de remblayage de la plate-forme.

\section{Q.5.9.}

\section{Mesures des déplacements}

Quatorze cibles de nivellement (N1 à N14), repérées à la figure 4 , ont été placées sur la plate-forme et sur le couronnement de la paroi. Les déplacements horizontaux de la paroi ont été mesurés par visées optiques sur des réglettes fixées au parement par des supports métalliques. La précision de ces mesures est de l'ordre du millimètre. Quatre tubes inclinométriques (I1 à I4) de $14 \mathrm{~m}$ de profondeur chacun ont été mis en place à partir de la plate-forme (Fig. 4). Trois sont implantés sur le profil d'étude (P 250) et un se situe au profil $\mathrm{P} 238$ à un mètre du couronnement de la paroi.

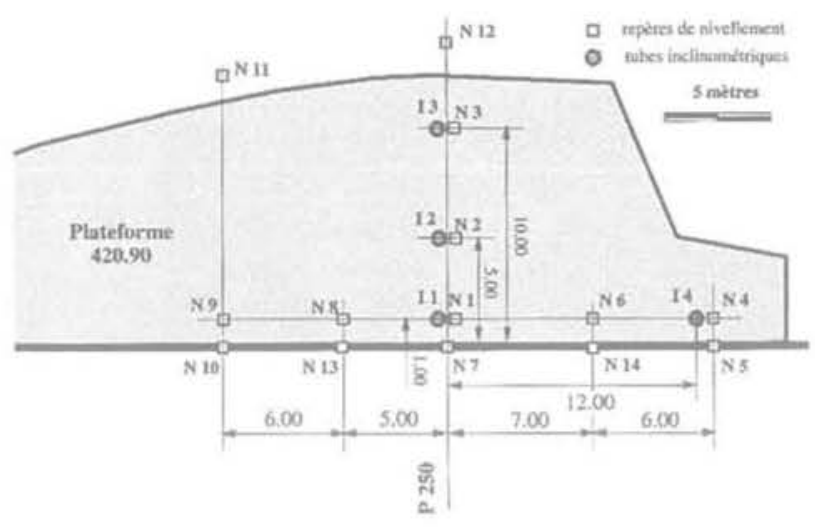

NG.4 Schéma d'implantation des repères de mesure en surface.

Reference marks for ground level measurements.

\section{Q.5.9.}

\section{Mesures des efforts mobilisés dans les barres}

Sur le profil d'étude ( $\mathrm{P}$ 250), les six rangs de clous ont été instrumentés à l'aide de jauges d'extensométrie collées sur les barres. Chaque barre est équipée d'une paire de jauges disposée tous les mètres linéaires. Les deux jauges sont placées de façon diamétralement opposée et le montage électronique est réalisé en demipont. On mesure ainsi la déformation axiale moyenne de la section de barre au point considéré, ce qui permet de calculer l'effort normal mobilisé. Pour assurer la précision des mesures, chaque barre a fait l'objet d'un étalonnage préalable en laboratoire.

\section{3}

\section{Observation} du comportement de l'ouvrage

\section{1}

\section{Déplacements de l'ouvrage}

L'évolution dans le temps des déplacements horizontaux et verticaux est respectivement représentée aux figures 5 et 6 , sur lesquelles l'avancement des travaux a été reporté. La situation précise des points de mesure est indiquée à la figure 4 .

Au cours de la réalisation de l'ouvrage, on observe une augmentation graduelle des déplacements horizontaux à mesure que la profondeur d'excavation s'accroit (Fig. 5). Le déplacement maximal en tête de la paroi (repère HN7) atteint $25 \mathrm{~mm}$ à l'issue de la dernière étape d'excavation (mesure du 16 mai). Les vitesses de déplacement qui étaient approximativement constantes pour les cinq premières étapes se réduisent considérablement lors de la dernière étape en raison de la présence de la couche raide à la base de l'ouvrage (alluvion ancienne).

Les déplacements verticaux sur la plate-forme subissent une évolution comparable à celle des déplacements horizontaux (Fig. 6). Le tassement maximal, mesuré sur le couronnement de la paroi (repère N7), est de $22 \mathrm{~mm}$. Il est obtenu à l'issue de la dernière étape 

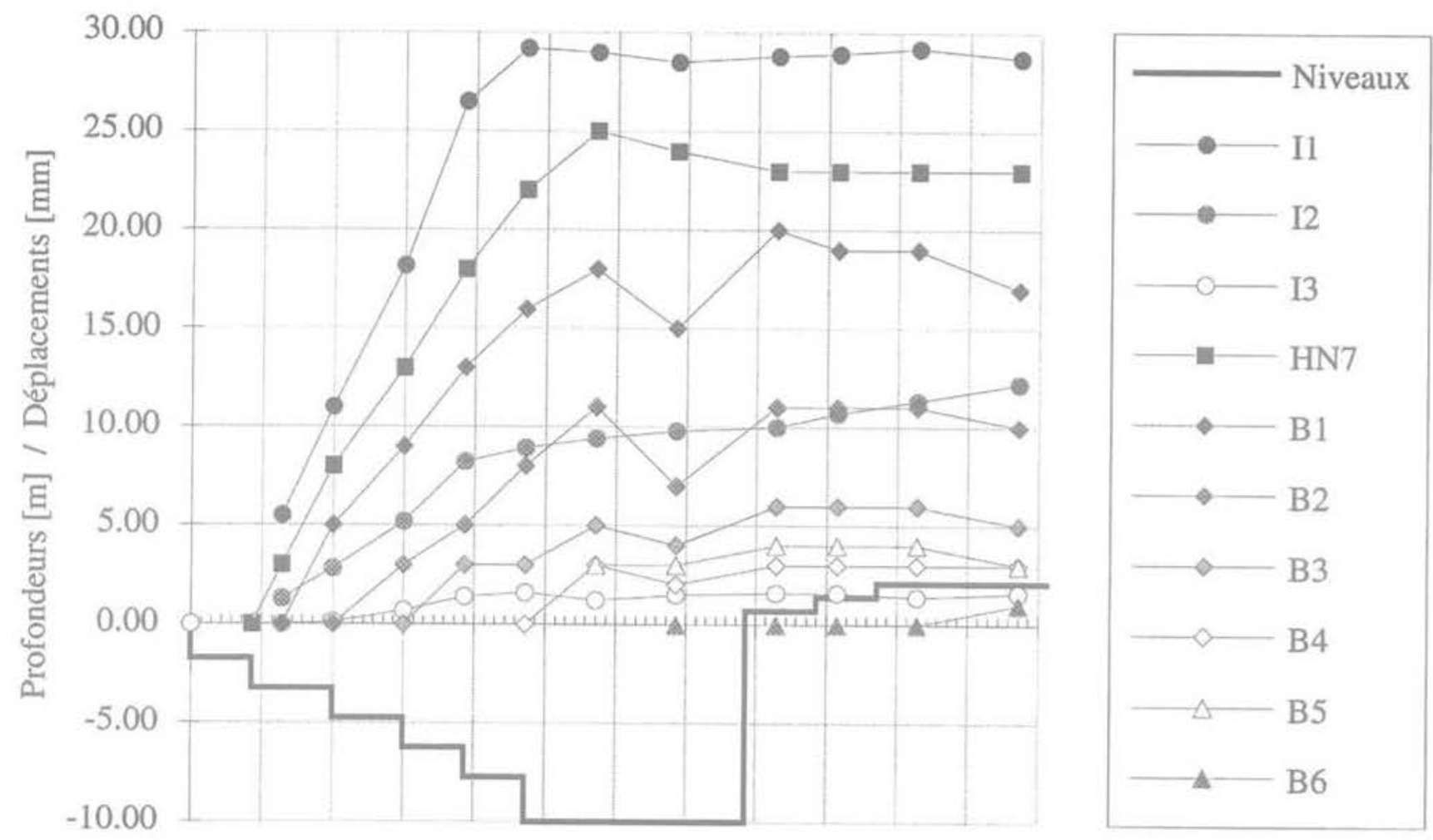

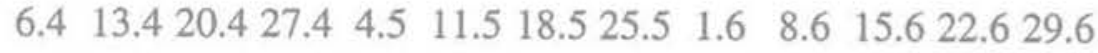 Dates}

F16.5 Évolution des déplacements horizontaux en fonction du temps. Evolution of the horizontal displacements with respect to time.

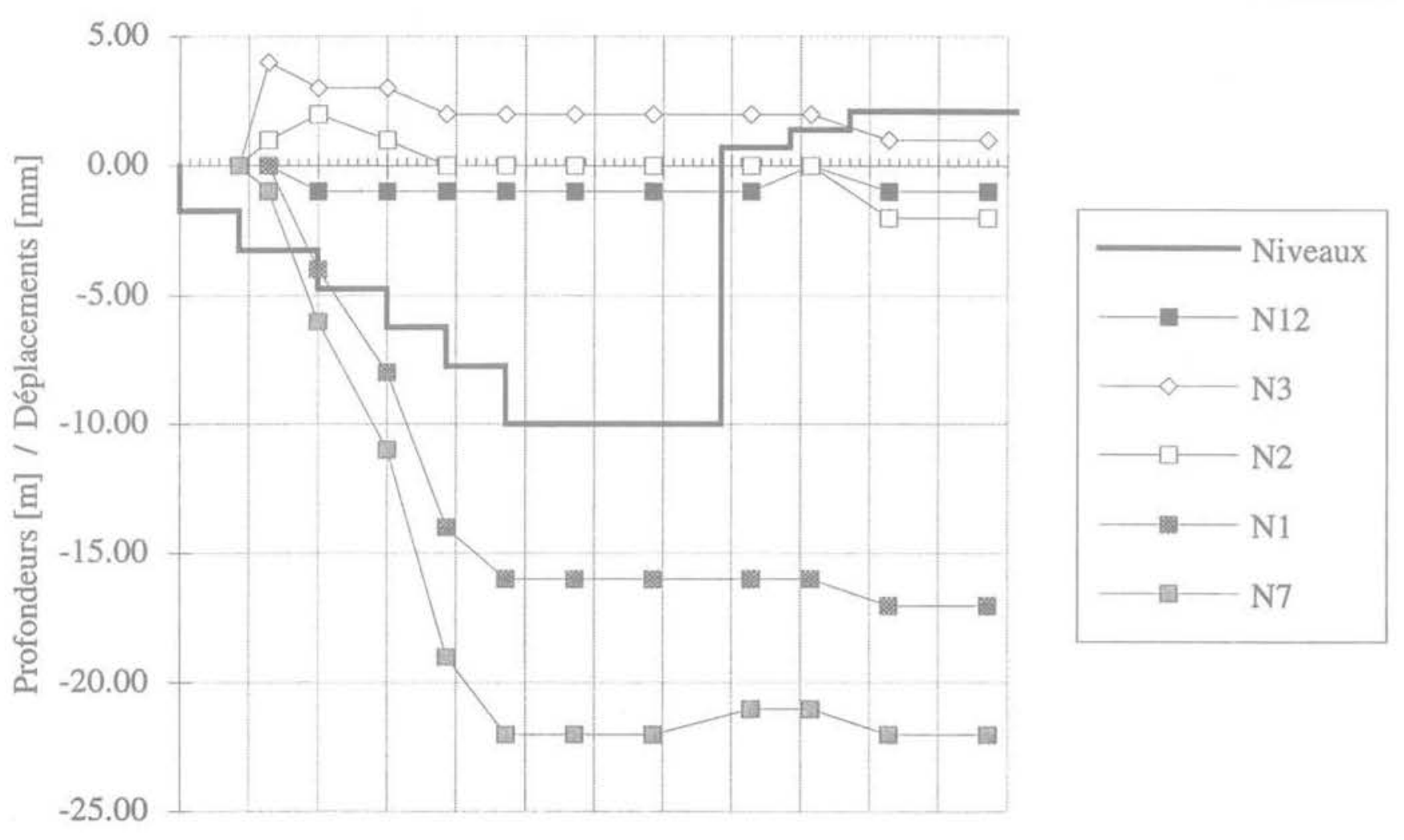

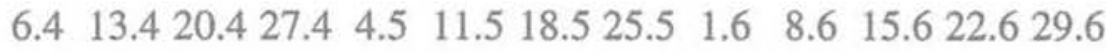

Dates

F1G.6 Évolution des déplacements verticaux en fonction du temps Evolution of the vertical displacements with respect to time. 
d'excavation. A mesure que l'on s'éloigne de la paroi, les tassements décroissent rapidement. A $1 \mathrm{~m}$ de la paroi, au droit de l'inclinomètre $I 1$ (repère N1), le tassement maximal n'est plus que de $16 \mathrm{~mm}$. A partir de $5 \mathrm{~m}$ (repères N2, N3), aucun tassement significatif n'a été enregistré.

Pour la période comprise entre la fin de l'excavation de l'ouvrage et le début du chargement (du 16 au 31 mai), les déplacements mesurés ne se sont pas accrus. On note au contraire un léger retour vers l'arrière de la partie supérieure de la paroi, tandis que les tassements se sont stabilisés. Le chargement de la plate-forme, appliqué entre le 31 mai et le 14 juin, n'a provoqué aucun accroissement des déplacements de l'ouvrage.

Les déplacements de la paroi et du massif de sol à l'issue de chacune des étapes de réalisation de l'ouvrage sont représentés sur les figures $9 \mathrm{a}$ à $9 \mathrm{~g}$ sur lesquelles les limites des couches géologiques ont également été reportées. Pour tenir compte des déplacements survenus avant la pose des reperes, une correction par extrapolation linéaire des valeurs lues sur les inclinomètres a été réalisée.

L'examen des profils inclinométriques montre que les déplacements décroissent avec la profondeur et avec la distance au parement de l'ouvrage. La décroissance des déplacements avec la profondeur est régulière et confirme l'absence d'une surface de glissement. On remarque cependant que, lors de la $3^{\mathrm{e}}$ étape d'excavation, le recoupement de la couche molle (moraine limoneuse) conduit à une légère perturbation dans le profil des déplacements enregistrés sur l'inclinomètre I1. Le déplacement maximal enregistré sur l'inclinomètre I1, qui est de $29 \mathrm{~mm}$ à la fin de l'excavation, s'atténue rapidement à mesure que l'on s'éloigne du parement.

Les déplacements mesurés sur la paroi elle-même décroissent aussi avec la profondeur. Cela indique un mouvement de translation associé à une légère rotation qui correspond à un basculement de la paroi vers l'aval. La perturbation observée sur le profil inclinométrique est encore plus évidente sur la paroi. Le déplacement à ce niveau (B3) est légèrement supérieur à celui du niveau supérieur (B2). Le dernier niveau (B6) n'indique aucun déplacement ce qui semble confirmer la raideur de la couche inférieure.

\section{Efforts mobilisés dans les barres}

L'évolution des efforts maximaux mobilisés dans chacune des barres en fonction du temps est représentée à la figure 7. Chaque barre se mobilise très rapidement lors des étapes d'excavation qui suivent sa mise en place : au-delà, l'effort dans la barre se stabilise. Durant la semaine de pause qui suit l'excavation, un léger déchargement des barres supérieures (B1 et B2), parfaitement conforme aux déplacements mesurés, est observé. Notons que les trois phases successives de

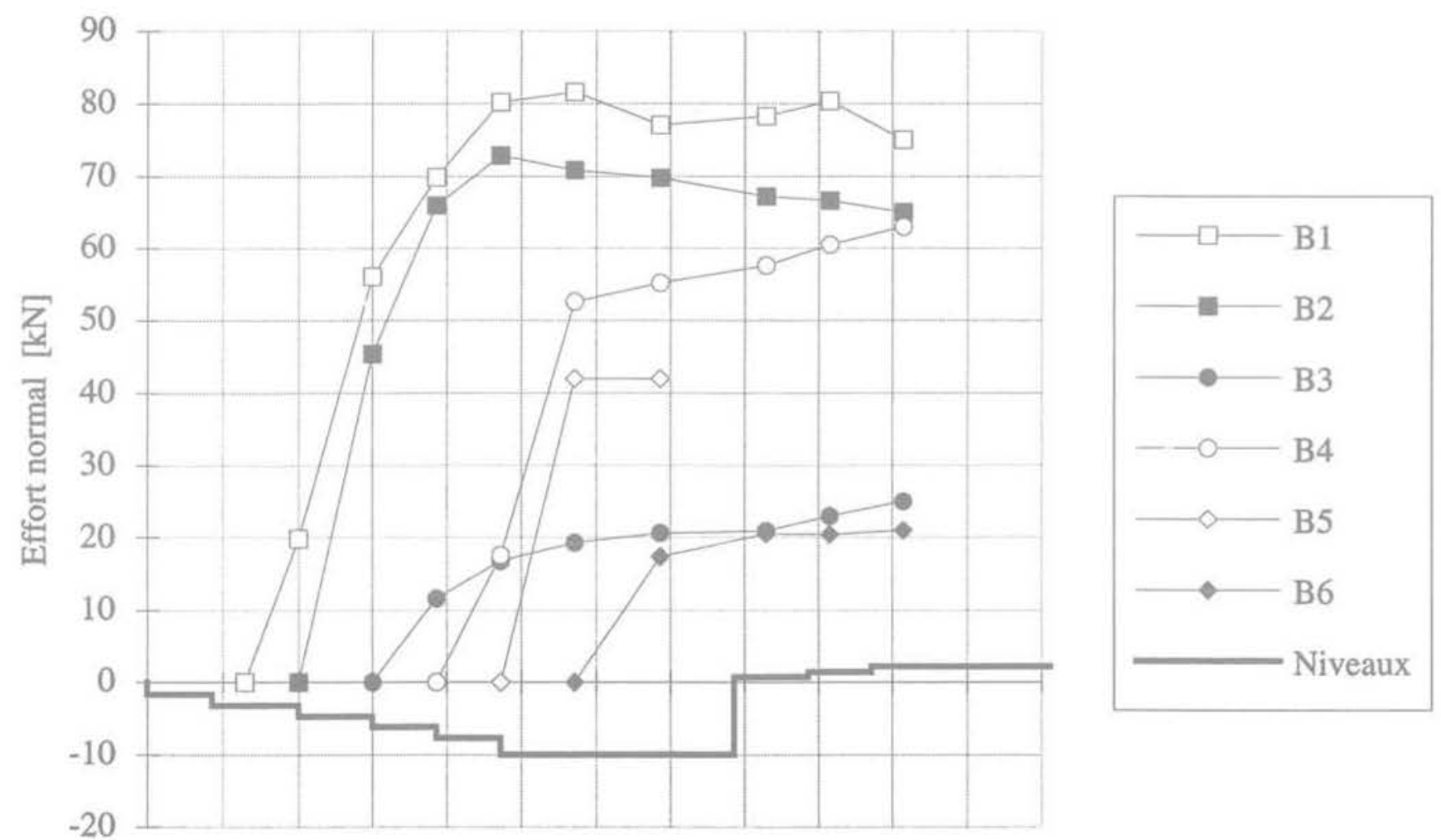

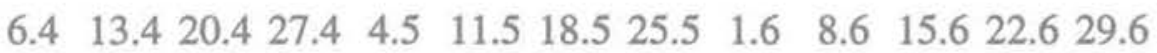

Dates

FG. 7 Évolution des efforts maximaux dans les barres en fonction du temps. Evolution of the maximal forces in the bolts with respect to time. 
chargement de la plate-forme, n'ont provoqué aucune augmentation substantielle des efforts dans les barres.

La distribution des efforts normaux mobilisés dans les barres au cours de l'excavation de l'ouvrage est indiquée aux figures $10 \mathrm{a}$ à $10 \mathrm{~g}$. Les limites des couches géologiques sont également reportées.

La mise en tension progressive de la première barre conduit lors de la troisième et de la quatrième étape d'excavation à une distribution approximativement constante de l'effort normal. A partir de la cinquième étape, on observe un point de traction maximal situé à $3,5 \mathrm{~m}$ du parement qui s'élève à $80 \mathrm{kN}$. Cet effort n'augmente plus lors des étapes d'excavation suivantes. Sur cette barre, un nombre important de jauges de déformation se sont détériorées réduisant à trois le nombre de jauges opérationnelles à la fin de l'essai. Notons cependant que les trois points de mesures restants encadrent l'effort normal maximal. La deuxième barre est rapidement mise en tension. L'effort normal dont la valeur maximale est obtenue à la cinquième étape d'excavation, atteint $73 \mathrm{kN}$. Cet effort décroît à mesure que l'on s'éloigne du parement. Sur cette barre, le point de tension maximal est situé à proximité du parement.

La troisième barre, mise en place dans la couche molle de moraine limoneuse, est extrêmement peu chargée comparativement aux deux précédentes. La distribution de l'effort normal dont le maximum atteint $19 \mathrm{kN}$ est approximativement constante le long de la barre. La quatrième barre, quant à elle, est mise en place partiellement dans la couche raide d'alluvions anciennes et partiellement dans la couche molle (moraine limoneuse). L'effort important (61 kN) enregistré à proximité du parement, chute brutalement au droit de l'interface de ces deux couches.

La cinquième barre est, elle aussi, relativement chargée. L'effort maximal atteinte $42 \mathrm{kN}$ à proximité du parement. Comme pour la précédente, une brutale chute de l'effort normal est observée à 1,5 m du parement. La sixième demeure relativement peu chargée ce qui semble logique compte tenu qu'aucune étape d'excavation n'a suivi sa mise en place.

\section{4}

\section{Synthèse des mesures}

L'analyse de l'auscultation de l'ouvrage montre que la quasi-totalité des déplacements se sont produits lors de la réalisation de l'ouvrage en raison du déconfinement consécutif à l'excavation. Le déplacement horizontal maximal en tête de paroi atteint environ $3 \%$ de la hauteur ce qui est comparable aux observations faites sur d'autres ouvrages (Clouterre, 1991). Les déplacements horizontaux et verticaux du massif de sol s'atténuent rapidement à mesure que l'on s'éloigne de la paroi. A $5 \mathrm{~m}$ du parement, au droit de l'inclinomètre I2, le déplacement horizontal mesuré en surface ne représente plus que $35 \%$ du déplacement de la tête de la paroi. A $10 \mathrm{~m}$, au droit de l'inclinomètre 13 , les déplacements horizontaux et verticaux sont insignifiants. L'application de la surcharge n'a produit aucun déplacement supplémentaire significatif.

La distribution des efforts dans les barres est conforme aux observations faites sur d'autres ouvrages. Les efforts maximaux sont localisés non loin du parement. Les valeurs maximales mesurées dans les barres supérieures, représentent environ $45 \%$ de leur résistance à la traction (respectivement $80 \mathrm{kN}$ et $180 \mathrm{kN})$. Le frottement mobilisé au contact barre-terrain ne dépasse pas $30 \mathrm{kN} / \mathrm{m}$, dans l'alluvion ancienne soit, $43 \%$ de la résistance à l'arrachement.

\section{4}

\section{Modélisation du comportement de l'ouvrage par la méthode des éléments finis}

\section{1}

\section{Introduction}

Une analyse numérique par la méthode des éléments finis a été entreprise pour modéliser le comportement de l'ouvrage.

Les calculs ont été réalisés à l'aide du programme EFEMER du Laboratoire de Mécanique des Roches de l'EPFL. Il s'agit d'un programme en élasto-plasticité qui permet de simuler une excavation en modifiant les caractéristiques des matériaux au cours du calcul (Dudt et Pellet, 1989). Les calculs ont été réalisés en déformations planes. Le maillage de l'ouvrage qui est représenté à la figure 8 , comprend 667 nouds qui définissent 616 éléments de surface. Les caractéristiques géomécaniques retenues après observation de la tenue des terrains lors de l'excavation de l'ouvrage sont résumées au tableau II.

Les clous sont représentés par des éléments-barres qui, dans le cas particulier, correspondent à des plaques de rigidité axiale équivalente. Les caractéristiques retenues sont les suivantes:

- module d'élasticité $\mathrm{E}=210000 \mathrm{MPA}$;

- section $\mathrm{A}=1,57.10^{-4} \mathrm{~m}^{2}$;

- effort à la rupture $\mathrm{F}=180 \mathrm{kN}$.

La paroi elle-même, constituée de béton projeté et de treillis d'armature, est représentée par des élémentspoutre dont les rigidités sont les suivantes:

- rigidité axiale $\mathrm{EA}=3,70,10^{6} \mathrm{kN}$;

- rigidité à la flexion $\mathrm{EI}=3,0.10^{4} \mathrm{kN} \cdot \mathrm{m}^{2}$.

Les calculs ont été réalisés pour les six étapes d'excavation et les trois phases de remblayage. Afin de représenter au mieux le processus de réalisation de l'ouvrage, chaque étape d'excavation a été divisée selon les sous-étapes suivantes:

- annulation des éléments excavés: 1 pas de calcul;

- activation des éléments de poutre (revêtement): 3 pas de calcul;

- activation des éléments de barre: calcul jusqu'à convergence.

\section{2}

\section{Comparaison des déplacements calculés et mesurés}

Les déplacements calculés lors de chacune des étapes sont représentés aux figures $9 \mathrm{a}$ à $9 \mathrm{~g}$. 

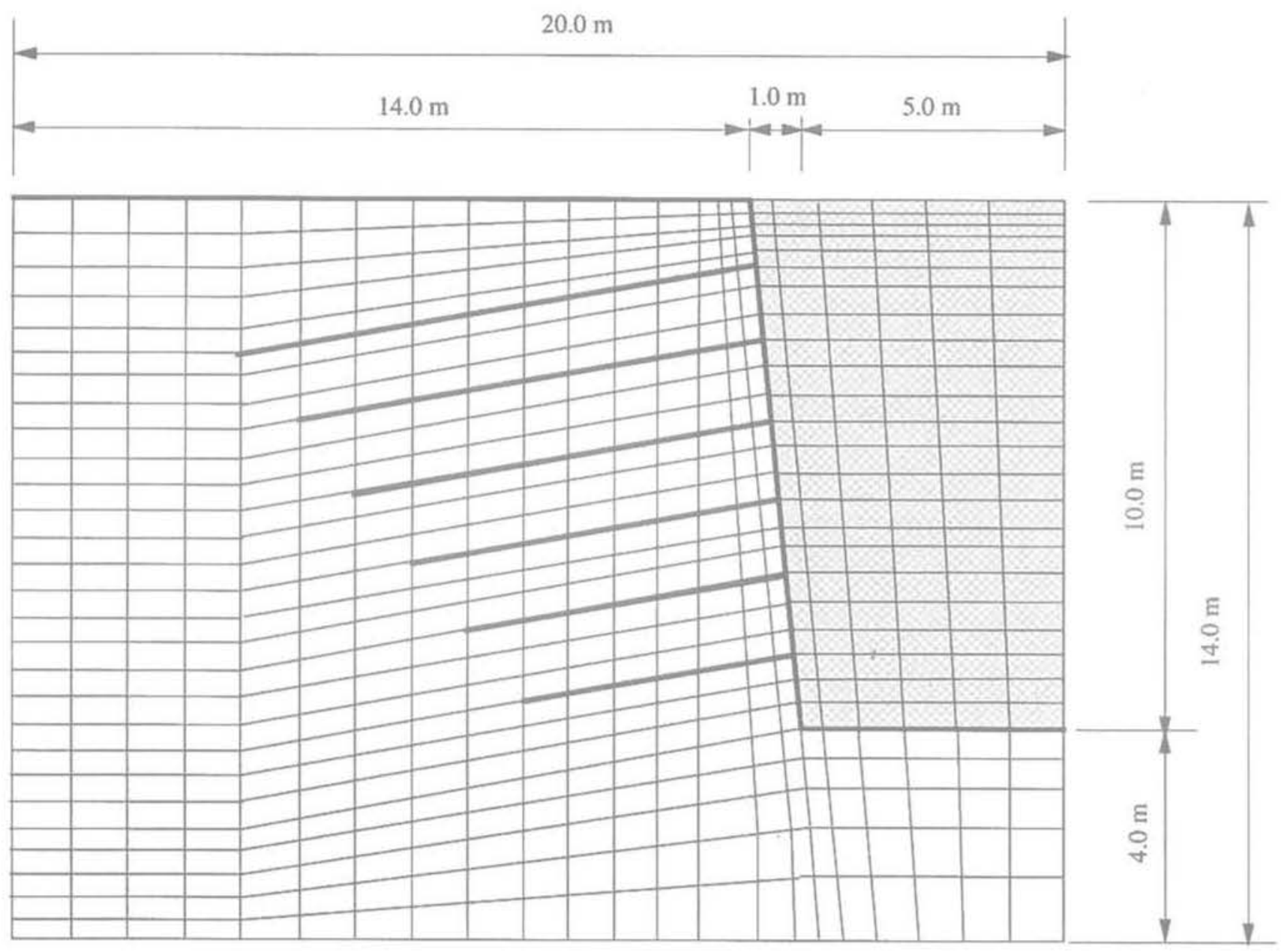

\section{Partie excavée}

FG.8 Maillage de l'ouvrage.

Finite element mesh

Lors des premières étapes, une bonne concordance entre valeurs mesurées et valeurs calculées est observée. A l'issue de la deuxième étape les déplacements calculés et mesurés en tête de paroi sont respectivement de 10 et $12 \mathrm{~mm}$. En arrière, au droit des inclinomètres $\mathrm{I} 1$ et 12 , situés respectivement à $1 \mathrm{~m}$ et à $5 \mathrm{~m}$ de la paroi, les déplacements calculés et mesurés en surface sont à peu près équivalents.
A mesure que l'excavation progresse, le déplacement calculé en tête devient inférieur à la valeur mesurée. L'écart maximal est observé à l'issue de la sixième étape d'excavation où le déplacement calculé est de $15 \mathrm{~mm}$ tandis que la valeur mesurée atteint $25 \mathrm{~mm}$. Sur la hauteur de la paroi les déplacements s'accordent mieux à l'exception de la zone située entre 6 et $8 \mathrm{~m}$ (barres B2 et B3) où les déplacements calculés sont net-

TABLEAUII Caractéristiques mécaniques des géomatériaux pour le calcul par éléments finis. Mechanical characteristics of geomaterials for finite element analysis.

\begin{tabular}{c|c|c|c|c} 
& $\begin{array}{c}\text { Poids volumique } \\
\gamma\left[\mathrm{kN} / \mathrm{m}^{3}\right]\end{array}$ & $\begin{array}{c}\text { Module d'élasticité } \\
\text { E [kPa] }\end{array}$ & $\begin{array}{c}\text { Cohésion } \\
\text { c [kPa] }\end{array}$ & $\begin{array}{c}\text { Angle de frottement } \\
\varnothing \text { degrés }\end{array}$ \\
\hline $\begin{array}{c}\text { Couche1 } \\
\text { Sable et graviers }\end{array}$ & 22,5 & 5000 & 5 & 33 \\
\hline $\begin{array}{c}\text { Couche2 } \\
\text { Moraine limoneuse }\end{array}$ & 23,5 & 12000 & 10 & 30 \\
\hline $\begin{array}{c}\text { Couche3 } \\
\text { Alluvionancienne }\end{array}$ & 24,0 & 30000 & 50 & 40 \\
\hline
\end{tabular}




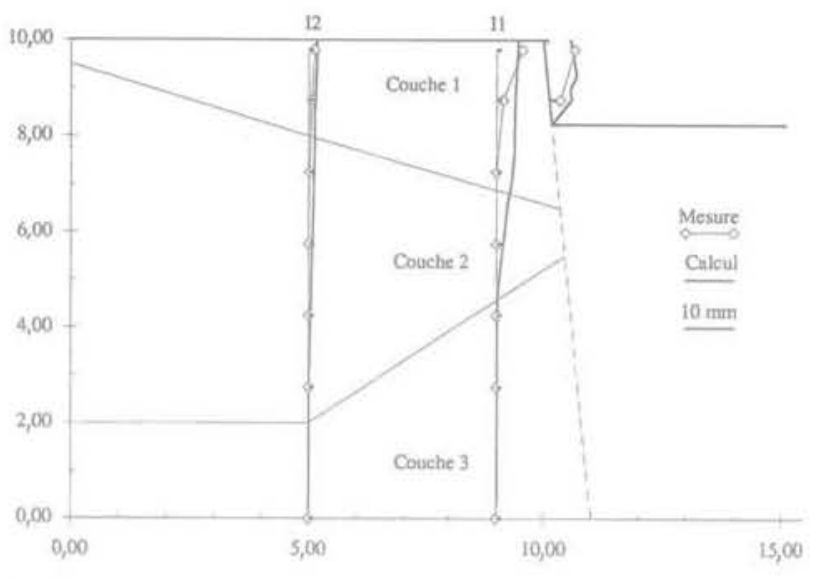

FGG.9a Déplacements calculés et mesurés à l'issue de la $1^{\text {re }}$ étape d'excavation.

Measured and calculated displacements after the "Ist excavation stage.

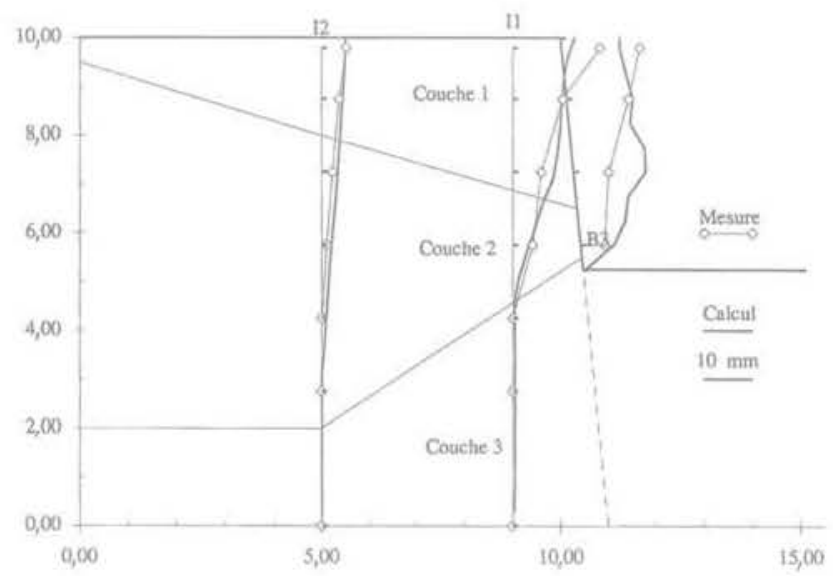

HG.9c Déplacements calculés et mesurés à l'issue de la $3^{e}$ étape d'excavation.

Measured and calculated displacements after the 3 rd excavation stage.

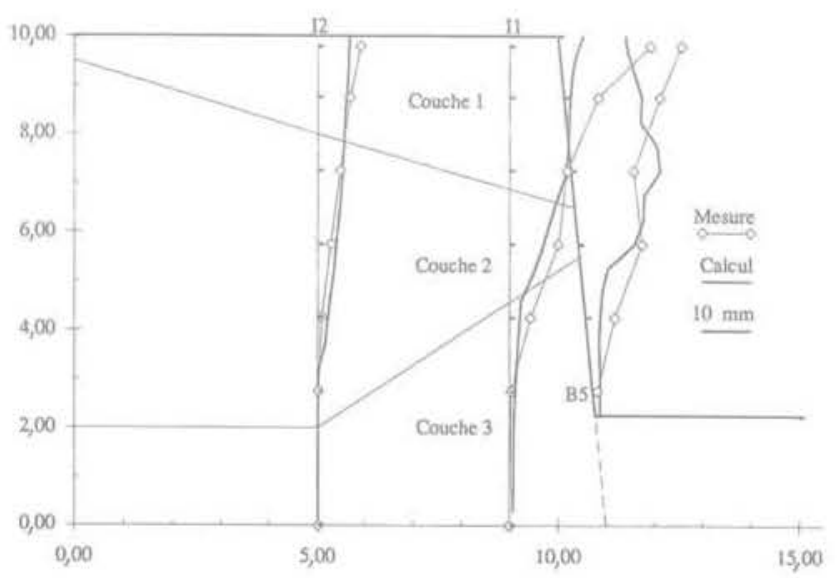

FiG. ge Déplacements calculés et mesurés à l'issue de la $5^{e}$ étape d'excavation.

Measured and calculated displacements after the 5 th excavation stage.

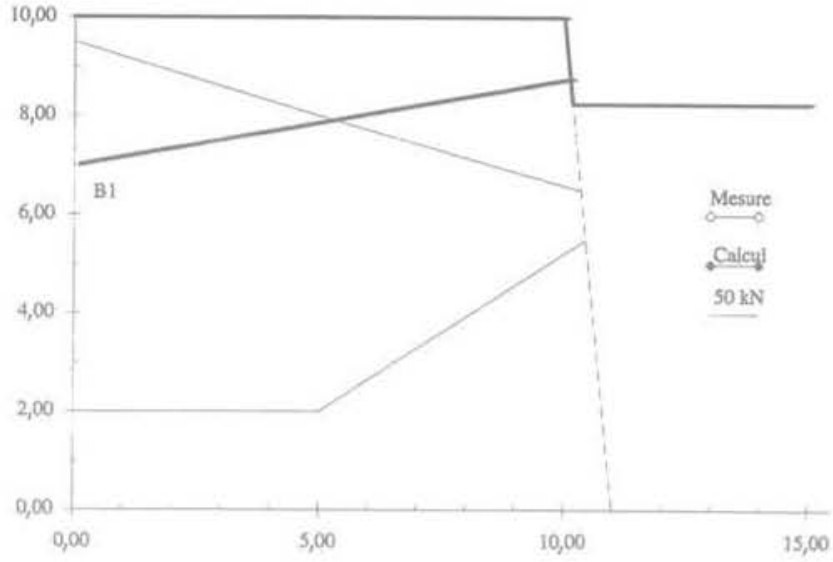

F0:90 Déplacements calculés et mesurés à l'issue de la $2^{\mathrm{e}}$ étape d'excavation.

Measured and calculated displacements after the 2nd excavation stage.

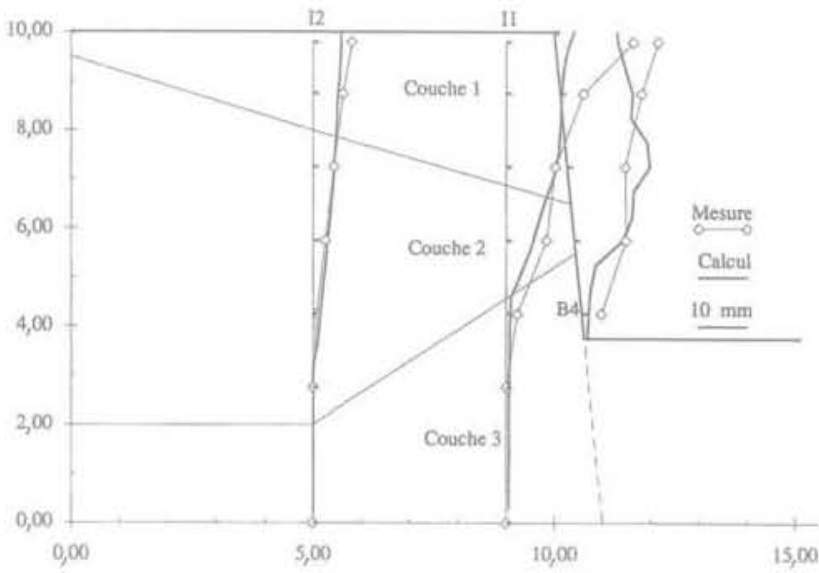

FiG.9d Déplacements calculés et mesurés à l'issue de la $4^{\circ}$ étape d'excavation.

Measured and calculated displacements after the 4th excavation stage.

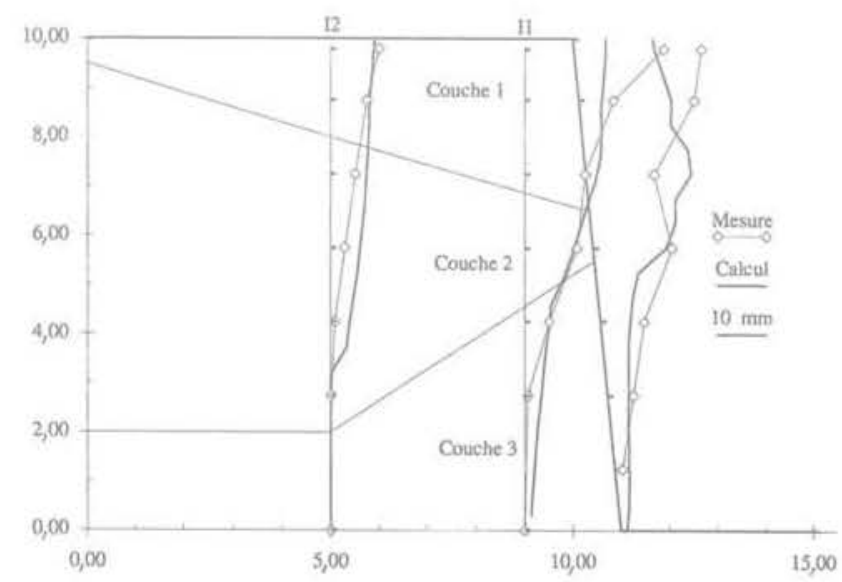

FIG. or Déplacements calculés et mesurés à l'issue de la $6^{e}$ étape d'excavation.

Measured and calculated displacements after the 6th excavation stage. 


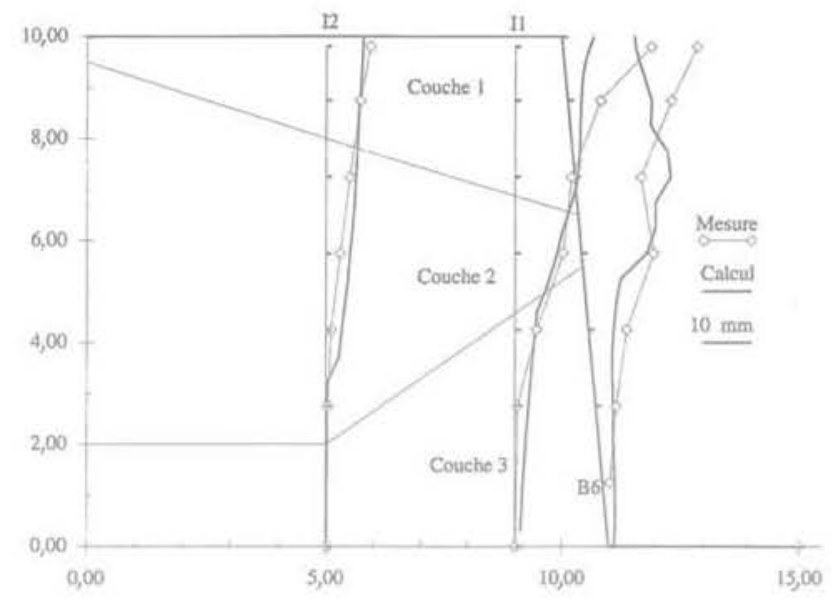

FG. $9 \mathrm{~g}$ Déplacements calculés et mesurés après l'application de la surcharge.

Measured and calculated displacements after the overloading. tement supérieurs aux valeurs mesurées. A la base de l'ouvrage, dans la couche raide d'alluvions anciennes, la concordance des déplacements est relativement bonne,

L'allure générale de la déformée calculée de la paroi montre des irrégularités dues à la flexion que subit chaque tronçon de paroi immédiatement après sa mise en place. Ces irrégularités sont particulièrement marquées lorsque l'ouvrage traverse la couche molle (repères B2 et B3).

La concordance des déplacements dans le massif de sol au droit de l'inclinomètre I1 est satisfaisante à l'exception du déplacement en surface dont la valeur mesurée à l'issue de la sixième étape d'excavation est de $29 \mathrm{~mm}$ contre $17 \mathrm{~mm}$ calculé. Plus en arrière sur l'inclinomètre 12, les profils de déplacements calculés et mesurés coïncident relativement bien. Le tassement mesuré de la tête de la paroi est de $22 \mathrm{~mm}$ alors que la valeur calculée est de $17 \mathrm{~mm}$. Comme les mesures l'ont mis en évidence, le calcul confirme que l'application de la surcharge ne provoque pas d'augmentation notable des déplacements.

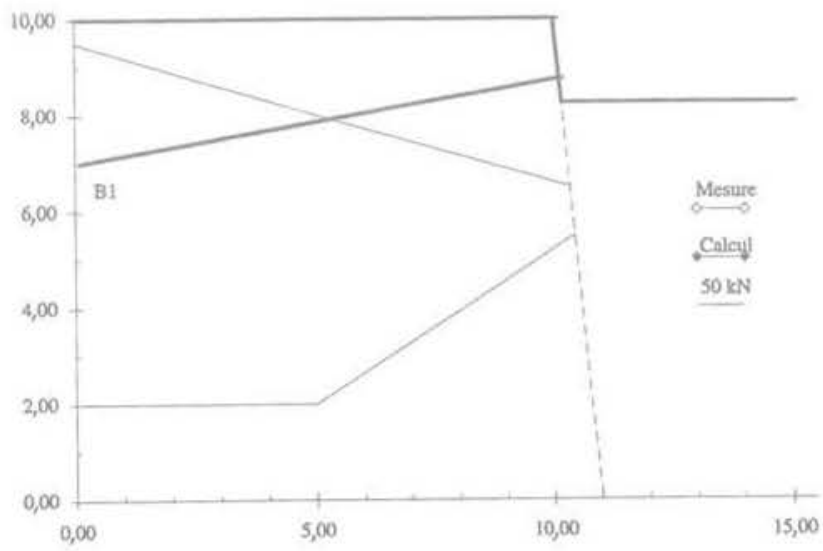

FG. 10a Efforts calculés et mesurés à l'issue de la $1^{\text {re }}$ étape d'excavation.

Measured and calculated forces after the 1st excavation stage.

\section{Comparaisons des efforts calculés et mesurés}

Les distributions des efforts mobilisés dans les barres lors des phases successives d'excavation sont représentées aux figures $10 \mathrm{a}$ à $10 \mathrm{~b}$.

Pour la première barre (B1) la valeur maximale de l'effort en fin d'excavation est nettement inférieure à la valeur mesurée. Lorsque la surcharge est appliquée, l'écart se réduit respectivement $80 \mathrm{kN}$ mesuré contre $58 \mathrm{kN}$ calculé. Le calcul indique un point d'effort maximal plus proche du parement que celui mis en évidence par les mesures.

Pour la deuxième barre (B2) l'écart entre les efforts maximaux mesurés et calculés est moindre. La valeur calculée à l'issue de la sixième étape d'excavation est de $53 \mathrm{kN}$ contre $71 \mathrm{kN}$ mesurée. L'application de la surcharge provoque une augmentation de l'effort calculé inférieur à celui observé pour la première barre. L'effort maximal est localisé à environ $1,5 \mathrm{~m}$ du parement.

La distribution des efforts calculés pour la troisième barre (B3) laisse apparaitre des efforts nettement supérieurs aux valeurs mesurées. Le calcul indique un effort maximal de $55 \mathrm{kN}$ après l'application de la surcharge, alors que la valeur mesurée n'est que de $23 \mathrm{kN}$. Cela pourrait être dù à une insuffisance d'adhérence liée à la mauvaise qualité de la couche traversée (couche 2) que le calcul ne prend pas en compte. On admet en effet, une adhérence parfaite entre la barre et le terrain.

A l'inverse de la barre précédente, les efforts calculés dans la quatrième barre (B4) sont inférieurs aux valeurs mesurées. L'effort maximal, enregistré à l'issue de la sixième étape, est localisé à $2 \mathrm{~m}$ du parement. Il est de $20 \mathrm{kN}$ alors que la mesure indique $53 \mathrm{kN}$.

La cinquième barre demeure relativement peu chargée. L'effort maximal calculé est de $20 \mathrm{kN}$ pour une valeur mesurée de $41 \mathrm{kN}$. La sixième barre mise en place après l'achèvement de l'excavation est, elle aussi, très peu sollicitée. On enregistre $21 \mathrm{kN}$ après l'application de la surcharge alors que le calcul indique $7 \mathrm{kN}$.

En résumé, les écarts entre efforts calculés et mesurés sont plus faibles pour les barres supérieures que

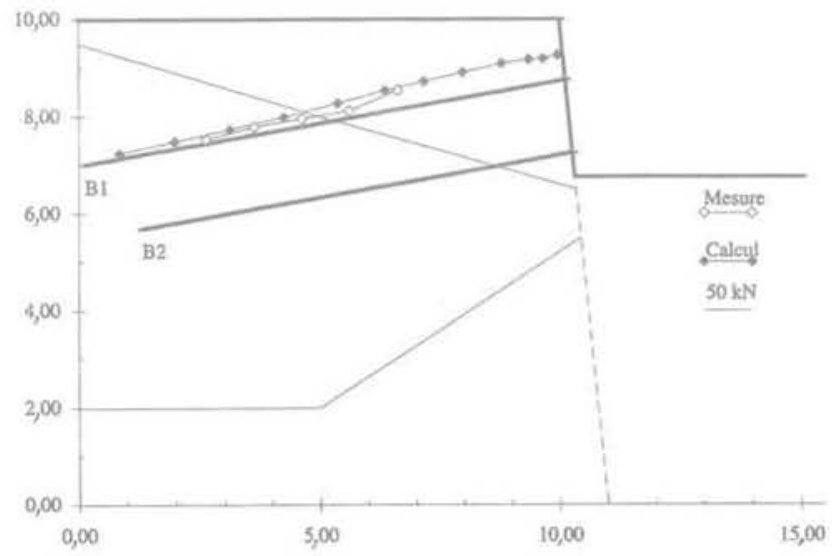

FG. 100 Efforts calculés et mesurés à l'issue de la $2^{e}$ étape d'excavation.

Measured and calculated forces after the 2nd excavation stage. 

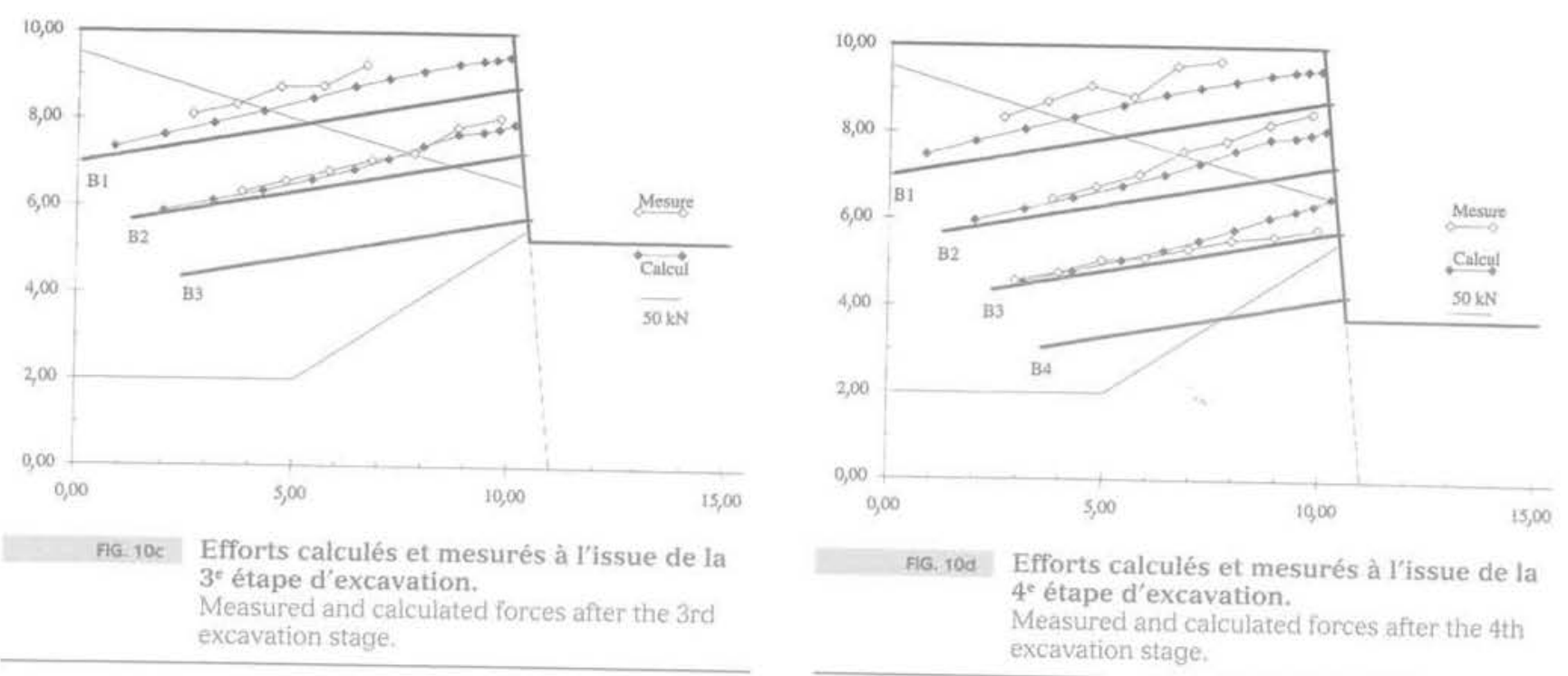

FiG. 10d Efforts calculés et mesurés à l'issue de la 4e étape d'excavation.

Measured and calculated forces after the 4 th excavation stage.
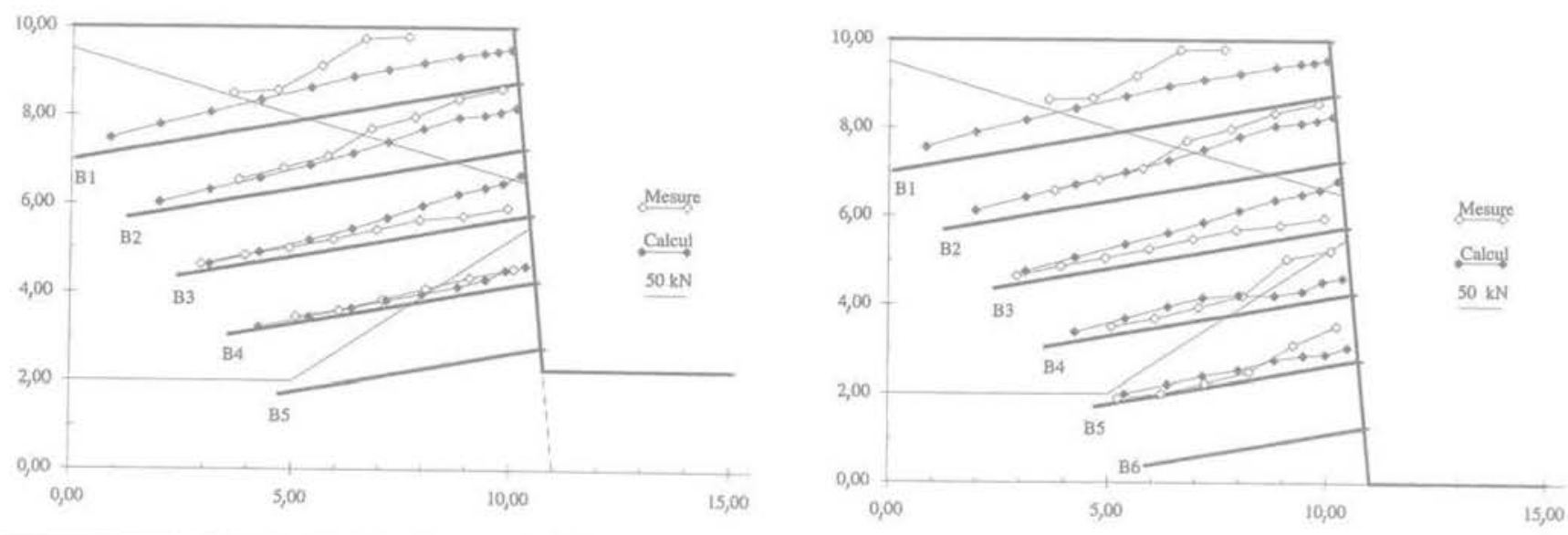

FIG. 10e Efforts calculés et mesurés à l'issue de la $5^{e}$ étape d'excavation.

Measured and calculated forces after the 5 th excavation stage.

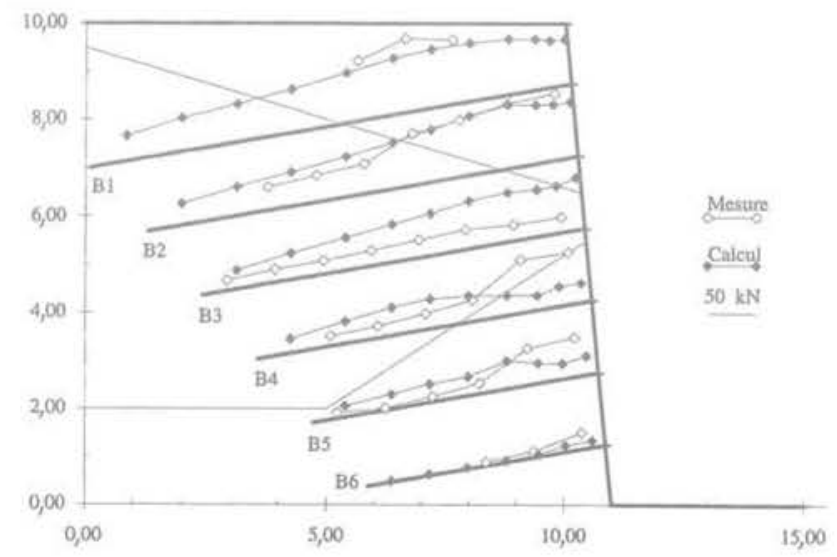

pour les barres inférieures. De manière générale, on observe des distributions d'efforts similaires indiquant une décroissance des efforts à mesure que l'on s'éloigne du parement.

\section{5}

\section{Conclusion}

L'analyse des résultats de l'instrumentation de l'ouvrage a permis de préciser son comportement mécanique au cours des différentes étapes de réalisation. Les déplacements horizontaux et verticaux sont concentrés à proximité du parement dans une zone qui s'étend sur une distance environ égale à la moitié de la hauteur de l'ouvrage. Au-delà les déplacements sont insignifiants. Les efforts maximaux mesurés dans les barres sont de l'ordre de la moitié de leur résistance à la traction et le frottement mobilisé est nettement infé- 
rieur au frottement limite. L'application d'une surcharge de hauteur équivalant au cinquième de la hauteur de l'ouvrage ne provoque aucune augmentation substantielle des déplacements de la paroi ni des efforts mobilisés dans les barres.

L'analyse numérique du comportement de louvrage par la méthode des éléments finis a montré qu'en dépit de la simplicité du modèle de comportement, on peut raisonnablement estimer les déplace- ments et les efforts dans les clous de ce type d'ouvrages.

\section{Remerciements}

L'Institut des sols, roches et fondations de l'Ecole polytechnique fédérale de Lausanne remercie l'Office fédéral des routes de la Confédération helvétique ainsi que le Département des travaux publics du Canton de Genève d'avoir accepté de financer ce projet.

\section{Bibliographie}

Anthoine A. - Une méthode pour le dimensionnement à la rupture des ouvrages en sols renforcés. Revue Française de Géotechnique, $n^{\circ} 50,1990$, pp. 5-17.

Blondeau F. Christiansen M. Guilloux A. Schlosser F, - Talren, Méthode de calcul des ouvrages en terre renforcée. Proc. Int. Symp. in Situ soil and Rock Reinforcement, Paris, Presses de J'ENPC, 1984, pp. 219-224.

De Buhan P. - Approche fondamentale du calcul à la rupture des ouvrages en sols renforcés. Thèse de doctorat d'état, Université Paris-VI, 1986.

Dudit J.-P., Pellet F. - EFEMER, Méthode des éléments finis en élasto-plasticité, présentation générale. ISRF-EPFL, 1989.
Gässler G. - Vernageite Geländesprünge Tragverhaiten und Standsichereit. Veröffentlichungen, Heft 108, Universität Fridericiana, Karsruhe, 1987.

Gudehus G. - Clouage des sols: règles de dimensionnement et leur vérification expérimentale. Revue Française de Géotechnique, $n^{\circ} 19,1981$, pp. 30-37.

Pellet F - Résistance et déformabilité des massifs rocheux stratifiés renforcés par ancrages passifs. Thèse de doctorat $n^{\circ} 1169$, Ecole polytechnique fédérale de Lausanne, 1993.

Pellet F, Egger P, Descoeudres F, - Étude du comportement d'une paroi expéri- mentale renforcée par clouage. Rapport R328, École polytechnique fédérale de Lausanne, 1994

Recommandations CLOUTERRE 1991 , pour la conception, le calcul, l'exécution et le contrôle des soutènements réalisés par clouage des sols. Paris, Presses de I'ENPC, $272 \mathrm{p}$.

Schlosser F., Unterreiner P., Plumelle C. Validation des méthodes de calcul de clouage par les expérimentations du projet national CLOUTERRE. Revue Française de Géotechnique, n 64, 1993, pp. $11-20$. 